\title{
CRP/Albumin Has a Promising Prospect as a New Biomarker for the Diagnosis of Periprosthetic Joint Infection
}

\author{
Weipeng Shi ${ }^{1,2}$ \\ Yingzhen Wang' \\ Xuan Zhao ${ }^{3}$ \\ Tengbo $\mathrm{Yu}^{\prime}$ \\ Tao Li' \\ 'Department of Orthopaedic Surgery, \\ The Affiliated Hospital of Qingdao \\ University, Qingdao, 266003, People's \\ Republic of China; ${ }^{2}$ Medical Department \\ of Qingdao University, Qingdao \\ Shandong, 26607I, People's Republic of \\ China; ${ }^{3}$ Department of Rheumatism and \\ Immunology, The Affiliated Hospital of \\ Jining Medical University, Jining, 272007, \\ People's Republic of China
}

Purpose: In the present study, we aimed to identify more effective biomarkers for the diagnosis of periprosthetic joint infection (PJI) by comparing the diagnostic ability of C-reactive protein (CRP)/albumin, systemic immune-inflammation index (SII), and existing biomarkers.

Methods: In this single-center retrospective analysis, 216 patients who underwent revision knee or hip arthroplasty from June 2013 to June 2021 and met the inclusion and exclusion criteria were analyzed, including 80 patients in the PJI group and 136 patients in the non-PJI group. The following information was collected for analysis, including CRP, fibrinogen, erythrocyte sedimentation rate (ESR), D-dimer, platelet count (PC), neutrophil count $(\mathrm{N})$, lymphocyte count (Lym), and albumin count on the second day of admission. The receiver operating characteristic (ROC) curve was used to compare the diagnostic ability of various biomarkers, and further subgroup analysis was carried out in the PJI group.

Results: Compared with the non-PJI group, CRP, fibrinogen, D-dimer, ESR, CRP/albumin, and SII in the PJI group were significantly increased $(\mathrm{P}<0.001)$, and their area under the curve (AUC) was 0.937, 0.878, 0.777, 0.914, 0.941, and 0.765, respectively. In the three subgroup analyses, there was no significant difference in appeal biomarker $(\mathrm{P}>0.05)$ based on PJI culture results, infection time, and infection site.

Conclusion: $\mathrm{CRP} /$ albumin had a promising prospect as a new biomarker for the diagnosis of PJI, while it had insufficient predictive ability in the PJI subgroup analysis. However, SII had insufficient ability to diagnose PJI. Therefore, SII was not suitable to be used as a diagnostic biomarker.

Keywords: periprosthetic joint infection, CRP/albumin, systemic immune-inflammation index, biomarker

\section{Introduction}

As one of the serious complications after total joint infection (TJA), periprosthetic joint infection (PJI) has a global incidence of about $5-20 \%,{ }^{1,2}$ and aseptic loosening also greatly contributes to the failure of TJA. $^{3}$ In 2011, the American Musculoskeletal Infection Society (MSIS) has proposed that the sinus communicating with the prosthesis or the affected joint with positive culture twice and the same bacteria can be used as the main standard of PJI, and the elevation of C-reactive protein (CRP) or erythrocyte sedimentation rate (ESR) is the secondary standard of diagnosis. ${ }^{4}$ In 2013, the International Consensus Meeting (ICM) has added new biomarkers, including leukocyte esterase, based on MSIS and set the diagnostic
Correspondence: Tao Li; Tengbo Yu Department of Orthopaedic Surgery, The Affiliated Hospital of Qingdao University, Qingdao, Shandong, People's Republic of China

Email litaodoctor@sina.com; ytb1970@I26.com 
threshold of acute and chronic infections. ${ }^{5}$ In 2018, Parvizi et al have summarized the original consensus and guidelines and formulated a new set of score-based diagnostic criteria for PJI, which has high sensitivity and specificity for the diagnosis of PJI. ${ }^{23}$

Routine plasma biomarkers, such as CRP and ESR, are widely used in clinical screening for infection because of their simple operation and economic effectiveness. However, both indicators will increase after TJA. Therefore, their diagnostic efficacy in the acute stage of infection is limited. Fibrinogen is one of the coagulation biomarkers for the traditional diagnosis of venous thrombosis and other diseases. Besides, fibrinogen is also an acute-phase player in the pathogenesis of infection. $\mathrm{Xu}$ et $\mathrm{al}^{6}$ have found that compared with other serum biomarkers, the level of plasma fibrinogen shows a better diagnostic effect before the revision of joint arthroplasty. Some recent investigations have shown that the CRP/albumin ratio is independently related to Gram-negative neonatal sepsis, and such a ratio can be used to predict Gramnegative bacteremia. ${ }^{7}$ Meanwhile, the CRP/albumin also exhibits good predictive value in acute pancreatitis and surgical site infection (SSI) after major abdominal surgery. ${ }^{8,9}$ The elevation of systemic immuneinflammation index (SII) value is mainly attributed to the increased neutrophil and platelet levels and the reduced lymphocyte levels, suggesting that the inflammatory response of patients is elevated, and the immune response is deteriorated. ${ }^{10}$ The diagnostic ability of the latter two indicators in infection has been confirmed, while there is no report on the performance in the diagnosis of PJI.

In the present study, we aimed to (1) compare whether CRP/albumin and SII were better than commonly used biomarkers in the diagnosis of PJI; and (2) explore the predictive ability of appeal biomarkers in culture-positive /negative PJI, acute/chronic PJI, knee/hip PJI, and cocci/ bacilli PJI.

\section{Materials and Methods}

This study was approved by the ethical committee of our institution, and experimental procedures were conducted following the declaration of Helsinki. All patients provided written informed consent before operation. A total of 271 patients who underwent revision of knee or hip arthroplasty in our institution from June 2013 to June 2021 were retrospectively analyzed in the present study. Patients diagnosed with a periprosthetic fracture or prosthesis dislocation $(n=28)$ were excluded. To exclude factors that might affect the results, patients with autoimmune diseases $(\mathrm{n}=5)$, malignant tumors $(\mathrm{n}=3)$, and hematological diseases, those using anticoagulant drugs for various reasons $(\mathrm{n}=16)$, and individuals with incomplete information $(n=3)$ were excluded from the study cohort. Finally, 216 patients were included in the research regimen. There were 80 patients in the PJI group and 136 patients in the non-PJI group, including patients with aseptic loosening, joint instability, and unexplained pain. All PJI patients met the diagnostic criteria of PJI introduced by the International Consensus Meeting (ICM) in 2018. ${ }^{23}$

\section{Data Extraction}

The basic information of all patients was acquired from the electronic medical record system of the institution, including age, location, time, symptoms, and complications. The patients were forbidden to eat and drink in the early morning of the day after hospitalization, and the venous blood samples were collected the next morning and sent to the laboratory within $1 \mathrm{~h}$. The levels of CRP, ESR, fibrinogen, D-dimer, platelet count (PC), neutrophils (N), lymphocytes (Lym), and albumin were analyzed. The SII was determined using the above-mentioned indicators with an equation as follows: $\mathrm{SII}=$ platelet count $*$ neutrophil count/lymphocyte count.

Biomarker testing methods: CRP: nephelometry immunoassay $(0 \sim 5.00 \mathrm{mg} / \mathrm{L})$; ESR: Westergren method (male $0 \sim 15 \mathrm{~mm} / \mathrm{h}$, female $0 \sim 30 \mathrm{~mm} / \mathrm{h}$ ); fibrinogen: Clauss method $(2.00 \sim 4.00 \mathrm{~g} / \mathrm{L})$; D-dimer: turbidimetric immunoassay $(0 \sim 500.00 \mathrm{ng} / \mathrm{mL})$; PC, N, Lym: flow cytometry method; albumin, colorimetry (Bromocresol Green, BCG).

Apart from biomarker testing, specimens of joint fluid/ pus and periprosthetic tissues obtained from patients with confirmed or suspected PJI were cultured under aerobic and anaerobic conditions, and histopathologic examination of bones or periprosthetic tissues were carried out.

\section{Statistical Analyses}

SPSS version 26.0 (IBM Inc., Armonk, NY, USA) was used for all statistical analyses. Normally distributed variables were analyzed using a $t$-test, and the Mann-Whitney $U$-test was adopted for the comparison between the two groups. All data were presented as mean \pm standard deviation. The count data between groups were compared with Chi-square, and descriptive statistics were presented as frequency or percentage. $\mathrm{P}$ less than 0.05 was considered statistically significant. The area under the curve (AUC), 95\% confidence interval (CI), and sensitivity and specificity of different markers 
Table I Demographic Characteristics of All Patients

\begin{tabular}{|c|c|c|c|}
\hline & $P J I^{a}(n=80)$ & Non-PJI $(n=\mid 36)$ & $P$ value \\
\hline Gender & & & 0.942 \\
\hline Male (\%) & $4 I(52.5)$ & $69(50.7)$ & \\
\hline Female (\%) & $39(47.5)$ & $67(49.3)$ & \\
\hline Age(y) & $64.6 I \pm 10.02$ & $65.12 \pm 9.75$ & 0.673 \\
\hline $\mathrm{BMI}^{\mathrm{b}}(\mathrm{kg} / \mathrm{m} 2)$ & $25.82 \pm 4.11$ & $24.70 \pm 3.49$ & 0.017 \\
\hline Joint & & & $<0.001$ \\
\hline Knee (\%) & $42(52.5)$ & $27(19.9)$ & \\
\hline Hip (\%) & $38(47.5)$ & $109(80.1)$ & \\
\hline
\end{tabular}

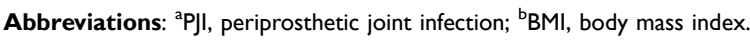

were determined using the working subject curve. The best cut-off value, positive predictive value (PPV), and negative predictive value (NPV) of markers were determined according to the Youden index. An AUC value of 0.900-1.000 was defined as excellent, 0.800-0.899 was good, 0.700-0.799 was fair, $0.600-0.699$ was poor, and 0.500-0.599 indicated no diagnostic ability. ${ }^{11}$

\section{Results}

\section{Demographic Characteristics of Patients}

No significant difference in terms of gender and age was detected among 216 included subjects $(\mathrm{P}>0.05)$. The body mass index (BMI) of the PJI group was remarkably greater compared with the non-PJI group $(\mathrm{P}<0.05)$, while more revision hip arthroplasty was found in the non-PJI group compared with the PJI group $(\mathrm{P}<0.001)$ (Table 1).

\section{Diagnostic Value of Different Biomarkers in PJI}

We compared the diagnostic abilities of several preoperative biomarkers (CRP, fibrinogen, D-dimer, and ESR), including their counts or ratios (CRP/albumin and SII) between the two groups. The biomarker count and ratio in the PJI group were remarkably greater compared with the non-PJI group ( $\mathrm{P}<$ 0.001) (Table 2). ROC (Figure 1) showed that the AUC ( $95 \%$ $\mathrm{CI})$ of the six biomarkers was 0.937 (0.903 0.972), 0.878 (0.831 0.925), 0.777 (0.712 0.842), 0.914 (0.877 0.952), $0.941(0.907 \sim 0.975)$, and $0.765(0.697 \sim 0.833)$, respectively. CRP/albumin, one of the main research objectives of this study, performed best, two traditional biomarkers (CRP and ESR) performed excellently, and two fibrinolytic markers (fibrinogen and D-dimer) performed well. However, the diagnostic value of another research index SII was fair, which performed worst among all indicators (Table 3).

Based on the Youden index, we further calculated the optimal cutoff value, sensitivity, specificity, PPV, and NPV of each biomarker (Table 3). The sensitivity of CRP was the highest $(86.3 \%)$, followed by CRP/albumin (83.8\%), while the sensitivity of SII was only $47.5 \%$. In terms of specificity, CRP/albumin and SII performed best, with a specificity of $94.9 \%$ and $92.6 \%$, respectively, and the specificity of D-dimer remained low (66.9\%). CRP/albumin performed well in both PPV and NPV $(90.4 \%$ and $90.2 \%$, respectively), while SII performed generally fair (79.2\% and $75.0 \%$, respectively) (Table 3 ).

\section{Predictive Value of Different Biomarkers in Subgroup Analysis}

In the present study, a subgroup analysis was carried out according to the culture results, including infection time, infection site, and types of infected bacteria of PJI patients. Among the 80 PJI patients, 54 were culture positive, and 26 were culture negative. No significant difference in biomarkers, except for ESR (45.71 vs $45.92 \mathrm{~mm} / \mathrm{h}$ ), was detected between the two subgroups, the other five biomarkers of culture-positive patients were only relatively greater compared with culture-negative patients (Table 4). The subjects infected within 3 months were defined as acute PJI, and those infected more than 3 months were regarded as chronic PJI. ${ }^{11}$ The results in the

Table 2 Comparison of Different Biomarkers Between PJI and Non-PJI

\begin{tabular}{|l|c|c|c|}
\hline & PJI $^{\mathbf{a}}(\mathbf{n}=\mathbf{8 0})$ & Non-PJI(n=136) & P value \\
\hline CRP $^{\mathrm{b}}(\mathrm{mg} / \mathrm{L})$ & $40.15 \pm 47.33$ & $3.57 \pm 4.96$ & $<0.001$ \\
Fibrinogen(g/L) & $4.72 \pm 1.32$ & $2.99 \pm 0.80$ & $<0.00 \mathrm{I}$ \\
D-dimer(ng/mL) & $1121.88 \pm 917.09$ & $488.05 \pm 266.92$ & $<0.001$ \\
$\operatorname{ESR}^{\mathrm{c}}(\mathrm{mm} / \mathrm{h})$ & $45.78 \pm 25.50$ & $12.88 \pm 10.52$ & $<0.001$ \\
CRP/albumin & $1.19 \pm 1.49$ & $0.09 \pm 0.15$ & $<0.001$ \\
SII $^{\text {d }}$ & $967.26 \pm 905.48$ & $445.69 \pm 342.21$ & $<0.001$ \\
\hline
\end{tabular}

Abbreviations: ${ }^{\mathrm{a}} \mathrm{PJI}$, periprosthetic joint infection; ${ }^{\mathrm{b}} \mathrm{CRP}, \mathrm{C}$-reactive protein; ${ }^{\mathrm{C}} \mathrm{ESR}$,erythrocyte sedimentation rate; ${ }^{\mathrm{d}} \mathrm{SIl}$,systemic immune-inflammation index. 

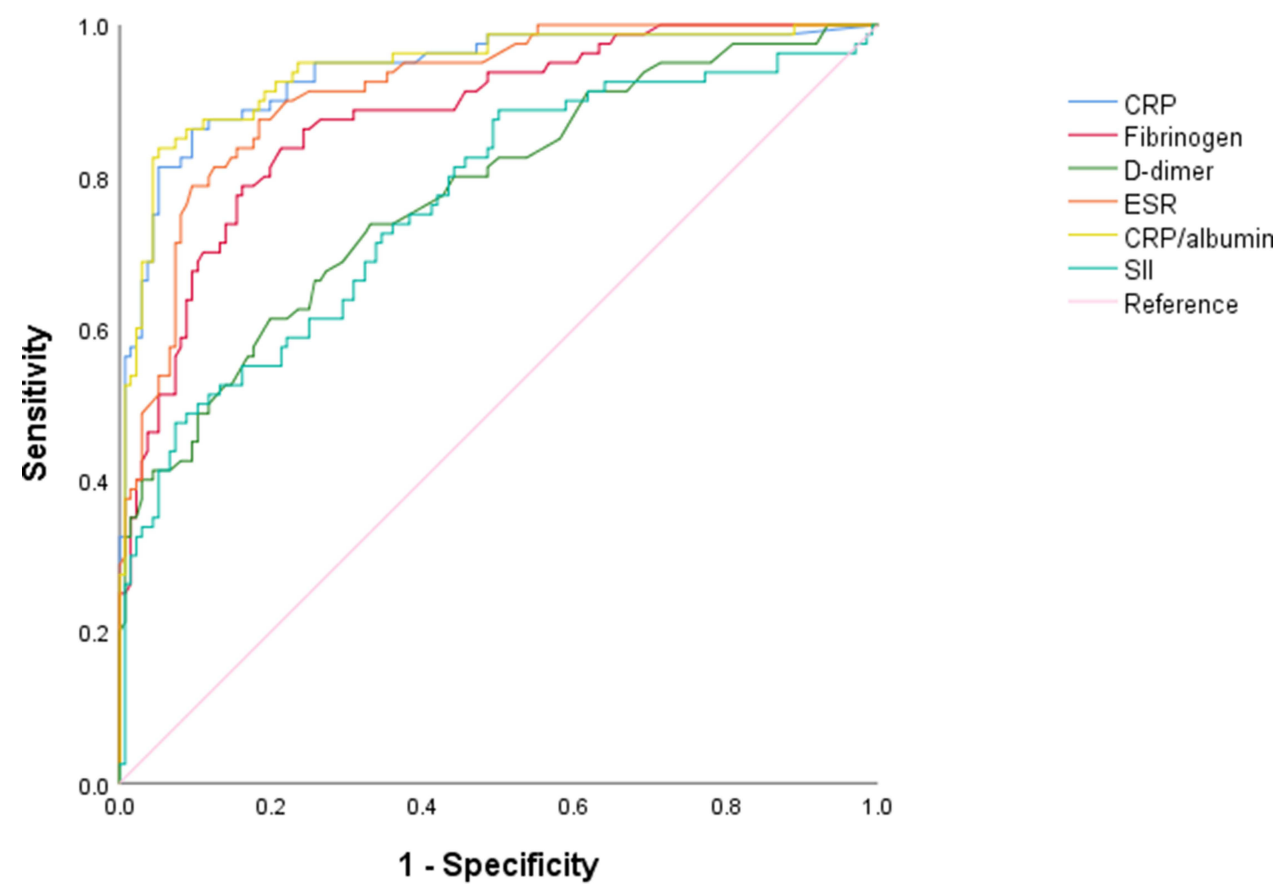

is the smallest, indicating that CRP / albumin has the best ability to diagnose PJI, while SII is the worst.

analysis of acute and chronic PJI, knee and hip PJI, cocci, and bacilli PJI subgroups were similar. Although relative differences were detected in different biomarkers among different subgroups, such differences remained nonstatistically meaningful $(\mathrm{P}>0.05)$ (Table 4$)$.

\section{Discussion}

We, for the first time, showed that CRP/albumin had a better diagnostic effect on the diagnosis of PJI compared with traditional biomarkers (CRP, ESR, and D-dimer). Although it could not provide better predictive ability in subgroup analysis, this biomarker still had good application prospects. Compared with CRP/albumin, SII had limited value in the diagnosis of PJI and could not be used as a diagnostic biomarker of PJI at present. Our results also showed that the existing biomarkers did not have good predictive value when distinguishing PJI from different culture results, different strains, different infection times, and different infection sites.

PJI is a disastrous result after total joint replacement. However, in the early stage after TJA, CRP, ESR, and other biomarkers are elevated because of the stress response of the body, ${ }^{12}$ and the chronic inflammatory response caused by the prosthetic device will also exhibit symptoms similar to $\mathrm{PJI},{ }^{13}$ such as pain and elevated inflammatory indicators. Therefore, how to quickly and accurately distinguish PJI and aseptic loosening has always been an issue in clinical practice. It also has important guiding significance for the following targeted treatment. However, at present, no effective gold standard

Table 3 Diagnostic Value of CRP, Fibrinogen, D-Dimer, ESR, CRP/Albumin, and SII for PJI

\begin{tabular}{|c|c|c|c|c|c|c|c|c|}
\hline & AUC & $95 \% \mathrm{Cl}$ & Youden Index & Optimal Cutoff Value & Sensitivity & Specificity & PPV $^{\mathbf{a}}$ & $\mathbf{N P V}^{\mathrm{b}}$ \\
\hline $\mathrm{CRP}^{\mathrm{C}}(\mathrm{mg} / \mathrm{L})$ & 0.937 & $(0.903,0.972)$ & 0.767 & 8.73 & $86.3 \%$ & $90.4 \%$ & $84.1 \%$ & $91.8 \%$ \\
\hline Fibrinogen(g/L) & 0.878 & $(0.831,0.925)$ & 0.626 & 3.68 & $78.7 \%$ & $83.8 \%$ & $77.6 \%$ & $87.0 \%$ \\
\hline D-dimer(ng/mL) & 0.777 & $(0.7 \mid 2,0.842)$ & 0.407 & 545 & $73.8 \%$ & $66.9 \%$ & $56.2 \%$ & $81.1 \%$ \\
\hline $\operatorname{ESR}^{\mathrm{d}}(\mathrm{mm} / \mathrm{h})$ & 0.914 & $(0.877,0.952)$ & 0.692 & 26.50 & $78.8 \%$ & $90.4 \%$ & $82.9 \%$ & $87.9 \%$ \\
\hline CRP/albumin & 0.941 & $(0.907,0.975)$ & 0.787 & 0.27 & $83.8 \%$ & $94.9 \%$ & $90.4 \%$ & $90.2 \%$ \\
\hline $\mathrm{SII}^{\mathrm{e}}$ & 0.765 & $(0.697,0.833)$ & 0.401 & 827.63 & $47.5 \%$ & $92.6 \%$ & $79.2 \%$ & $75.0 \%$ \\
\hline
\end{tabular}

Abbreviations: ${ }^{\mathrm{a} P P V}$, positive predictive value; ${ }^{\mathrm{b}} \mathrm{NPV}$, negative predictive value; ${ }^{\mathrm{C} C R P}, \mathrm{C}$-reactive protein; ${ }^{\mathrm{d} E S R}$,erythrocyte sedimentation rate; ${ }^{\mathrm{e}} \mathrm{SIl}$, systemic immuneinflammation index. 


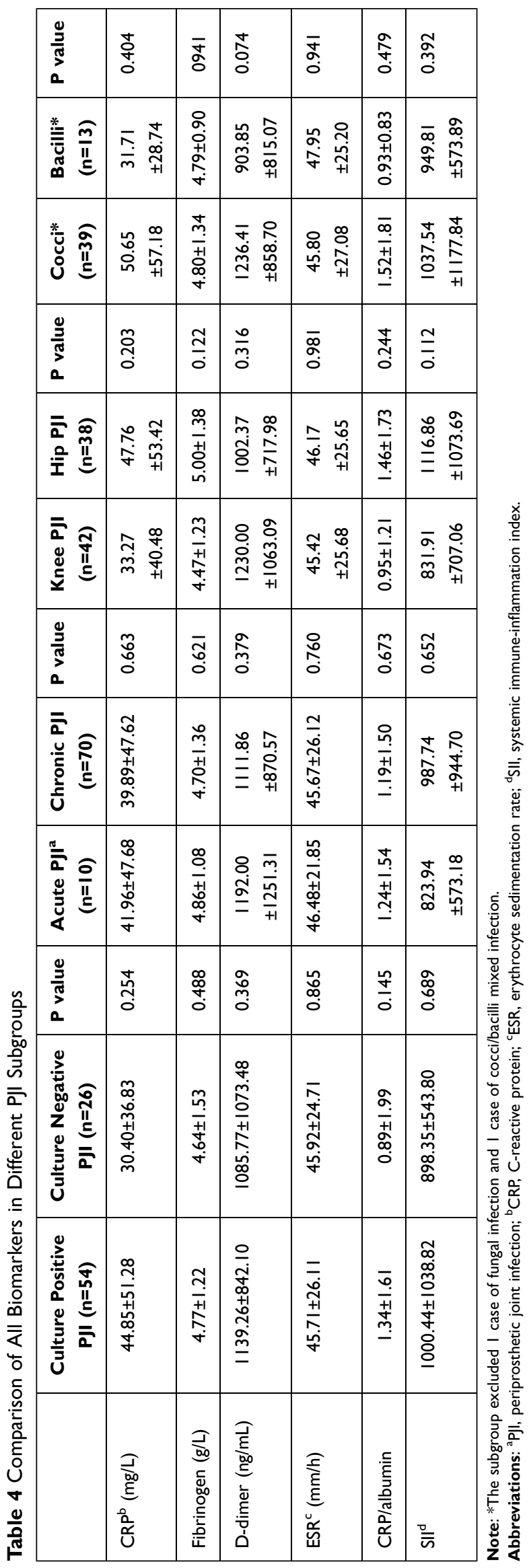

has not been found. Recent studies have reported the potential of fibrinolytic indicators (including fibrinogen, FDP, and D-dimer), ${ }^{6,13,14}$ platelet-related indicators (such as $\mathrm{PC}, \mathrm{PC} /$ volume), ${ }^{15}$ and protein-related biomarkers (such as albumin, globulin, and albumin-globulin ratio) in the diagnosis of PJI. ${ }^{11}$ Recent non-orthopedic reports have shown that CRP/albumin and SII have good predictive values in SSI, and prognosis of department of general surgery and neonatal sepsis..$^{7-10}$

In our study, the specificity of CRP reached $90.4 \%$, which was considered to be related to the exclusion of patients with autoimmune diseases and other complications. However, in some studies, CRP shows low sensitivity, which may be attributed to that the pathogenic bacteria are low-virulence microorganisms, which form a biofilm to protect the pathogen against the host immune system and reduce the host immune response, leading to reduced release of inflammatory markers. The CRP concentration in serum is low. ${ }^{17}$ In addition, a study believes that CRP cannot be adopted as the diagnostic standard in lowvirulence pathogen infection and chronic PJI. ${ }^{18}$ Other similar studies have also reported that it is highly possible to have a false-negative result in the diagnosis of PJI based on serum CRP. ${ }^{16}$ Many influencing factors were excluded from this study. Therefore, the results of CRP were better than previous studies. However, the patients' conditions are complicated in clinical practice, and CRP has certain limitations in predicting infections. The research on albumin in the existing literature mainly focuses on evaluating the nutritional status of the body. Some studies have reported that the albumin content may alter during infection, ${ }^{19}$ while Cao et al have shown that no apparent correlation exists between albumin and PJI. ${ }^{11}$ We considered that it might be related to the long half-life of serum albumin, and its concentration would not change dramatically with the acute inflammatory reaction.

Based on the limitation of CRP and albumin in diagnosing infection alone, scholars have taken CRP/albumin as a new biomarker to study its ability to predict infection in recent years. ${ }^{7,9} \mathrm{CRP}$ and albumin are biomarkers for routine admission detection, which are economical and convenient. In addition, CRP/albumin, as a ratio, can reduce the error caused by a single factor of CRP or albumin.

Therefore, we, for the first time, introduced this biomarker into the diagnostic approach of PJI and aimed to explore its new value in the field of joint infection. We found that the AUC (0.941) of CRP/albumin was better compared with CRP (0.937), ESR (0.914), and fibrinogen 
(0.878), and the best cut-off value was 0.27. According to the calculation results of the Youden index, CRP/albumin was as sensitive as CRP, while it had the highest specificity. The above-mentioned data showed that CRP/albumin had a better comprehensive ability compared with traditional biomarkers in the diagnosis of PJI. Given the different treatment schemes for different types of PJI, especially the different types of antibiotics used by cocci and bacilli, this study conducted a subgroup analysis of PJI patients. However, in the subgroup analysis, there was no significant difference among all biomarkers including CRP/albumin of culture-positive and culture-negative PJI groups, acute and chronic PJI groups, knee and hip PJI groups, and cocci and bacilli PJI groups, indicating that the predictive ability of biomarkers was insufficient. Wang et al have also reported that D-dimer and fibrinogen cannot distinguish culture positive and negative $\mathrm{PJI} .{ }^{20}$

SII is first developed as a tool to predict the prognosis of liver cancer based on lymphocytes, neutrophils, and PC. Its principle is mainly realized through the functions of three types of cells and their interactions with tumor cells. PC decrease, neutrophil increase, or lymphocyte decrease suggests that the inflammatory state is elevated, and the immune state is exacerbated. ${ }^{10}$ At present, the ratio of neutrophils/lymphocytes (NLR) has been shown to have the ability to diagnose PJI, ${ }^{21,22}$ and PC/lymphocytes (PLR) is markedly associated with early PJI. ${ }^{22}$ Therefore, whether SII constructed by these three kinds of cells has the same diagnostic value has aroused our interest. In the present study, we, for the first time, designed an experiment to verify the ability of SII to diagnose PJI.

However, SII did not achieve the expected effect in this study. Through AUC, we found that the diagnostic ability of SII was general (0.765), and the performance of SII was the worst among the six biomarkers, which was significantly lower compared with other traditional biomarkers, such as CRP and ESR, or our latest marker CRP/albumin. The sensitivity of SII was only $47.5 \%$, and its specificity was $92.6 \%$, indicating that it could only be an exclusion index rather than a diagnostic index for the diagnosis of PJI. Further analysis indicated that SII had no significant difference in all subgroups. In conclusion, SII could not diagnose PJI at present.

There are several limitations in our current work. The single-center retrospective study is inevitably affected by the existing data, and the inherent bias is difficult to avoid. The sample size is small, especially there are only 10 cases of acute PJI. The findings of the subgroup analysis need to be verified using large samples of acute PJI. In our present study, all patients with complications were excluded, resulting in reduced reliability of conclusions. Whether the new biomarker is also applicable to these patients will be verified in the next research.

\section{Conclusions}

Collectively, CRP/albumin was a simple and economical biomarker with excellent diagnostic ability. It could be used as a routine prediction tool for the clinical diagnosis of PJI. Moreover, SII had a poor diagnostic ability for PJI, and it was not suitable to be used as a diagnostic biomarker at present.

\section{Data Sharing Statement}

All the case details can publish when approval is obtained from the Institutional Review Board (IRB) Affiliated Hospital of Qingdao University.

\section{Ethics Approval and Consent to Participate}

This study was approved by the Institutional Review Board (IRB) Affiliated Hospital of Qingdao University. All personal details were erased before analysis to cover patient data confidentiality and comply with the Declaration of Helsinki.

\section{Consent for Publication}

All authors agreed on the final version of the manuscript.

\section{Funding}

Partial financial support was received from the National Natural Science Foundation of China; Grant number: 81802151; Shandong Province Natural Science Foundation; Grant number: ZR2019MH012; China Postdoctoral Science Foundation; Grant number: 2018M642616; Qingdao Applied Foundational Research Youth Project; Grant number: 19-6-2-55-cg.

\section{Disclosure}

The authors declare they have no conflicts of interest.

\section{References}

1. Delanois RE, Mistry JB, Gwam CU, Mohamed NS, Choksi US, Mont MA. Current epidemiology of revision total knee arthroplasty in the United States. J Arthroplasty. 2017;32(9):2663-2668. doi:10.1016/j.arth.2017.03.066

2. Koh CK, Zeng I, Ravi S, Zhu M, Vince KG, Young SW. Periprosthetic joint infection is the main cause of failure for modern knee arthroplasty: an analysis of 11,134 knees. Clin Orthop Relat Res. 2017;475 (9):2194-2201. doi:10.1007/s11999-017-5396-4 
3. Cherian JJ, Jauregui JJ, Banerjee S, Pierce T, Mont MA. What host factors affect aseptic loosening after THA and TKA? Clin Orthop Relat Res. 2015;473(8):2700-2709. doi:10.1007/s11999-015-4220-2

4. Workgroup Convened by the Musculoskeletal Infection Society. New definition for periprosthetic joint infection. J Arthroplasty. 2011;26 (8):1136-1138. doi:10.1016/j.arth.2011.09.026

5. Parvizi J, Gehrke T, Chen AF. Proceedings of the international consensus on periprosthetic joint infection. Bone Joint J. 2013;95-B (11):1450-1452. doi:10.1302/0301-620X.95B11.33135

6. Xu H, Xie J, Yang J, Chen G, Huang Q, Pei F. Plasma fibrinogen and platelet count are referable tools for diagnosing periprosthetic joint infection: a single-center retrospective cohort study. J Arthroplasty. 2020;35(5):1361-1367. doi:10.1016/j.arth.2019.12.015

7. Gunes H, Yurttutan S, Cobanusagi M, Doganer A. CRP/albumin ratio: a promising marker of gram-negative bacteremia in late-onset neonatal sepsis. Turk Arch Pediatr. 2021;56(1):32-36. doi:10.14744/ TurkPediatriArs.2020.99076

8. Kaplan M, Ates I, Akpinar MY, et al. Predictive value of C-reactive protein/albumin ratio in acute pancreatitis. Hepatobiliary Pancreat Dis Int. 2017;16(4):424-430. doi:10.1016/S1499-3872(17)60007-9

9. Donlon NE, Mohan H, Free R, et al. Predictive value of CRP/ albumin ratio in major abdominal surgery. Ir J Med Sci. 2020;189 (4):1465-1470. doi:10.1007/s11845-020-02238-y

10. Hu B, Yang XR, Xu Y, et al. Systemic immune-inflammation index predicts prognosis of patients after curative resection for hepatocellular carcinoma. Clin Cancer Res. 2014;20(23):6212-6222. doi:10.1158/1078-0432.CCR-14-0442

11. Wang H, Zhou H, Jiang R, Qian Z, Wang F, Cao L. Globulin, the albumin-to-globulin ratio, and fibrinogen perform well in the diagnosis of Periprosthetic joint infection. BMC Musculoskelet Disord. 2021;22(1):583. doi:10.1186/s12891-021-04463-7

12. Honsawek S, Deepaisarnsakul B, Tanavalee A, et al. Relationship of serum IL-6, C-reactive protein, erythrocyte sedimentation rate, and knee skin temperature after total knee arthroplasty: a prospective study. Int Orthop. 2011;35(1):31-35. doi:10.1007/s00264-010-0973-0

13. Xu H, Shang G, Wang Y, Xiang S. Plasma fibrinogen is a reliable marker for diagnosing periprosthetic joint infection and determining the timing of second-stage revision. Int $J$ Infect Dis. 2021;108:220-225. doi:10.1016/j.ijid.2021.05.068

14. Bin G, Xinxin Y, Fan L, Shenghong W, Yayi X. Serum fibrinogen test performs well for the diagnosis of periprosthetic joint infection. J Arthroplasty. 2020;35(9):2607-2612. doi:10.1016/j.arth.2020.04.081
15. Paziuk T, Rondon AJ, Goswami K, Tan TL, Parvizi J. A novel adjunct indicator of periprosthetic joint infection: platelet count and mean platelet volume. J Arthroplasty. 2020;35(3):836-839. doi:10.1016/j.arth.2019.10.012

16. Akgun D, Muller M, Perka C, Winkler T. The serum level of C-reactive protein alone cannot be used for the diagnosis of prosthetic joint infections, especially in those caused by organisms of low virulence. Bone Joint J. 2018;100-B(11):1482-1486. doi:10.1302/ 0301-620X.100B11.BJJ-2018-0514.R1

17. Zimmerli W, Moser C. Pathogenesis and treatment concepts of orthopaedic biofilm infections. FEMS Immunol Med Microbiol. 2012;65(2):158-168. doi:10.1111/j.1574-695X.2012.00938.x

18. Perez-Prieto D, Portillo ME, Puig-Verdie L, et al. C-reactive protein may misdiagnose prosthetic joint infections, particularly chronic and low-grade infections. Int Orthop. 2017;41(7):1315-1319. doi:10.1007/s00264-017-3430-5

19. Ruot B, Papet I, Bechereau F, et al. Increased albumin plasma efflux contributes to hypoalbuminemia only during early phase of sepsis in rats. Am J Physiol Regul Integr Comp Physiol. 2003;284(3):R707R713. doi:10.1152/ajpregu.00483.2002

20. Wang Y, Li Y, Qiao L, Sun S. Comparison of a comprehensive set of fibrinolytic markers with C-reactive protein and erythrocyte sedimentation rate for the diagnosis of periprosthetic joint infection. $J \quad$ Arthroplasty. 2020;35(9):2613-2618. doi:10.1016/j.arth.202 0.04 .096

21. Yu BZ, Fu J, Chai W, Hao LB, Chen JY. Neutrophil to lymphocyte ratio as a predictor for diagnosis of early Periprosthetic joint infection. BMC Musculoskelet Disord. 2020;21(1):706. doi:10.1186/ s12891-020-03704-5

22. Zhao G, Chen J, Wang J, et al. Predictive values of the postoperative neutrophil-to-lymphocyte ratio, platelet-to-lymphocyte ratio, and lymphocyte-to-monocyte ratio for the diagnosis of early periprosthetic joint infections: a preliminary study. J Orthop Surg Res. 2020;15(1):571. doi:10.1186/s13018-020-02107-5

23. Parvizi J, Tan TL, Goswami K, et al. The 2018 definition of periprosthetic hip and knee infection: an evidence-based and validated criteria. J Arthroplasty. 2018;33:1309-1314.e2. doi:10.1016/j. arth.2018.02.078
Infection and Drug Resistance

\section{Publish your work in this journal}

Infection and Drug Resistance is an international, peer-reviewed openaccess journal that focuses on the optimal treatment of infection (bacterial, fungal and viral) and the development and institution of preventive strategies to minimize the development and spread of resistance. The journal is specifically concerned with the epidemiology of

\section{Dovepress}

antibiotic resistance and the mechanisms of resistance development and diffusion in both hospitals and the community. The manuscript management system is completely online and includes a very quick and fair peerreview system, which is all easy to use. Visit http://www.dovepress.com/ testimonials.php to read real quotes from published authors. 